\title{
Nucleic Acid Drug Delivery and Targeting
}

\author{
Yoshinobu Takakura
}

Received: 2 February 2011 / Accepted: 3 February 2011 / Published online: 24 February 2011

(C) Springer Science+Business Media, LLC 2011

Nucleic acids, DNA and RNA, are endogenous materials which encode genetic information. Advances in molecular biology in terms of understanding gene expression and regulation mechanisms together with great progress in biotechnology have generated a new paradigm in which these nucleic acids are used as medicines. Gene therapy has a relatively long history since the first clinical trial was carried out in 1990. One major strategy has been the use of viral vectors encoding a therapeutic gene for ex vivo and in vivo gene therapies. However, a significant contribution from pharmaceutical scientists has been expected for the development of nonviral gene delivery systems based on plasmid DNA (naked plasmids, lipoplexes, polyplexes, etc.) as safer vectors. Unfortunately, gene therapy has not been very successful in general, although many clinical protocols using viral and nonviral vectors have been evaluated.

On the other hand, great attention has been paid to another type of nucleic acid drug, siRNA (small interfering RNA). RNA interference (RNAi) is a potent and specific posttranscriptional gene silencing event in which double-stranded RNA degrades target mRNA. Since the discovery that siRNA, a class of synthetic short double-stranded RNA, can induce RNAi in mammalian cells, it has been expected that siRNA will be used in vivo as a therapeutic agent for the treatment of a variety of diseases. Needless to say, the establishment of delivery systems for siRNA is key to the success of this novel type of RNAi-based therapy, and this subject has been a major interest for many pharmaceutical scientists.

Pharmaceutical Research has been a platform offering recent advances in nucleic acid drug delivery and targeting studies performed mainly by pharmaceutical scientists. This theme issue includes one review article and six original research

Y. Takakura $(\triangle)$

Graduate School of Pharmaceutical Sciences, Kyoto University

Sakyo-ku

Kyoto 606-850I, Japan

e-mail: takakura@pharm.kyoto-u.ac.jp papers in order to provide readers of Pharmaceutical Research with the latest information on progress in this research field.

In the review article, Bonamassa et al. focus on the applications of hydrodynamic gene delivery in pharmaceutical research, such as to use hydrodynamic delivery to study the transcriptional regulation of CYP enzymes, to establish animal models for viral infections, and to analyze gene drug discovery and gene function. Three original articles also focus on gene delivery. Takiguchi et al. report the importance of the generation of reactive oxygen species as the molecular mechanism for activation/reactivation of transgene expression after hydrodynamic injection. Negishi et al. describe the delivery of the bFGF gene into ischemic muscle using novel bubble liposomes in combination with ultrasound exposure. Niu et al. report the delivery of a plasmid coding for a specific cytotoxic fusion peptide, p14ARF-TAT, using an FGF2-targeted cationic lipid vector for in vivo cancer therapy.

This theme issue also features research papers devoted to other types of nucleic acid drugs. Schaffert et al. describe the in vivo antitumor effect mediated by the targeted polyplex-based delivery of poly(I:G), doublestranded RNA with interferon-inducing activity, using EGF-polyethylene glycol-linear polyethylenimine. Dinh et al. report the lipoplex-based delivery of short doublestranded oligonucleotide, $\mathrm{NF \kappa B}$ decoy, for the treatment of osteoclastogenesis using mannosylated cationic liposomes via inhibition of proinflammatory cytokine production from macrophages. Yang et al. describe the delivery of TGF- $\beta 1$ pri-miRNA-producing plasmids driven by a specific promoter for the treatment of liver fibrosis via RNAimediated gene silencing.

We hope that the present issue will provide useful information on the most recent progress in the field of nucleic acid drug delivery research and will be helpful to the pharmaceutical scientists involved in this particular research field. Finally, we express sincere gratitude to the contributors to this issue for their efforts. 


\section{INTERVIEW WITH YOSHINOBU TAKAKURA}

What do you think holds the key to your success as a pharmaceutical scientist?

I have been fortunate to have dedicated mentors, good colleagues and students throughout my academic carrier. I have been encouraged by these people, which has enabled me to work hard and continuously as a pharmaceutical scientist.

What do you consider to be your key research accomplishments?

I have been involved in macromolecular drug delivery research in the field of biopharmaceutics. Macromolecular drugs involve drug conjugates with macromolecular carriers, protein drugs, and nucleic acid drugs, such as antisense oligonucleotides and plasmid DNA-expressing therapeutic proteins and short interfering RNA (siRNA). My earlier research accomplishments include a series of pharmacokinetic studies on model macromolecules and the development of delivery systems for antitumor drugmacromolecule conjugates and protein drugs based on pharmacokinetic analysis. I also studied the pharmacokinetics of nucleic acids in an earlier period, which provided the basis for my later gene delivery work. My most recent research accomplishments involve the establishment of delivery systems and strategies for various types of nucleic acid drugs in order to optimize non-viral gene therapy and RNAi-based therapy.

What was the turning point in your career?

I have not changed my gear so much as a pharmaceutical scientist since I started my professional research carrier as an associate professor at Kyoto University. Although I might have made small changes in direction after promotions, my basic research philosophy and interests have remained unchanged.

Which individuals have most influenced your research career?

First of all, my first mentor was the late Professor Hitoshi Sezaki. I started my research career as an undergraduate and graduate student in Professor Sezaki's lab at Kyoto University. Professor Mitsuru Hashida has also been my important mentor. I learned everything, what science is about, what a pharmaceutical scientist is, and many other things from both these mentors. The third person is Professor Ronald Borchardt at Kansas University. I think I became an "international" pharmaceutical scientist after working as a postdoctoral student in the lab of this American mentor.
Pharmaceutical scientists are faced with the dilemma of having to publish in biomedical or basic science journals. Does this mean cutting-edge science will not likely be featured in journals like Pharmaceutical Research?

I don't think so. Pharmaceutical Research and basic science journals have different basic characteristics. Although the definition of "cutting-edge" is difficult, cutting-edge pharmaceutical science will be featured.

Where is the field of nucleic acid drug delivery and targeting going, and how do the articles in the theme section fill the gap?

Design and delivery are important aspects for nucleic acid drugs. Of course, the development of novel delivery systems, novel carriers and delivery strategies, is an important issue for pharmaceutical scientists. However, I think the importance of the design of nucleic acid drugs has been increasing. How to select a target and how to regulate it, what type of nucleic acid to be selected, and how to design a nucleic acid drug are all critical for this research field. I hope the articles in the theme section will cover all these topics.

What are the challenges for nucleic acid-based therapeutics, and how can these challenges be overcome?

The main challenges involve design and delivery. I believe pharmaceutical scientists should be able to meet these challenges themselves or in collaboration with scientists in other research fields. It is difficult for pharmaceutical scientists to take a leading role in the development of classical small drugs where organic and medicinal chemists are mostly involved in design and synthesis. On the other hand, pharmaceutical scientists could make a significant contribution to the development of nucleic acid-based therapeutics.

What is the key to developing successful collaborative relationships among scientists at your university?

Good interactions between scientists with different scientific backgrounds in terms of their individual experience and technological skills are key. Integrated knowledge and technologies will synergistically enhance the potential for collaborative research.

\section{What is your philosophy of educating graduate students?}

Any student may be able to change into a creative pharmaceutical scientist during his/her research activities in an academic lab. I think being able to provide them with the opportunities to enjoy science is the most important thing that will allow them to find their 
potential. I believe frequent scientific discussions with them will stimulate their research activities and increase their ability to change.

\section{What are the challenges facing the pharmaceutical sciences?}

Drug delivery will continue to be the main challenge. Significant progress has been made with classical small drugs, peptide and protein drugs, and nucleic acid drugs. The development of novel delivery systems with a higher potential will be a critical issue for pharmaceutical scientists, especially for nucleic acid drugs.

\section{What is the place for collaboration with industry in academia?}

Any research effort in basic pharmaceutical sciences could be extended to collaboration with industry. Pharmaceutical scientists in academia are generally interested in pursuing fundamental findings for drug development and clinical applications. On the other hand, pharmaceutical scientists in industry are closer to the actual everyday problems and have the experience and network skills to solve these problems. Collaborative research between academia and industry will stimulate both and lead to fruitful results when it works well.

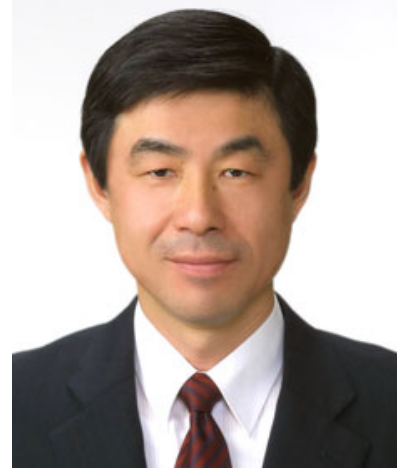

Dr. Yoshinobu Takakura has been a Professor at the Graduate School of Pharmaceutical Sciences, Kyoto University, since 1997. He received his Ph.D. in Pharmaceutical Sciences from Kyoto University in 1986. He was a postdoctoral fellow at the Department of Pharmaceutical Chemistry, University of Kansas, in Professor Ronald T. Borchardt's lab. He has conducted a series of studies in the field of macromolecular drug delivery in the field of biopharmaceutics. His earlier research accomplishments include basic pharmacokinetic studies on model macromolecules and development of delivery systems for antitumor drugmacromolecule conjugates and protein drugs based on the pharmacokinetic analysis. His most recent research interest involves nucleic acid drug delivery for optimization of gene therapy, DNA vaccination, and RNAibased therapies. He has published 243 peer-reviewed original research articles, 33 review articles, and 18 book chapters. He is a "highly cited researcher" in Pharmacology on the website ISIHighlyCited.com. He is an Executive Editor of Advanced Drug Delivery Reviews and a member of the Editorial Boards of Pharmaceutical Research, European Journal of Pharmaceutical Sciences, Journal of Biochemistry, and Drug Metabolism and Pharmacokinetics. His honors include Young Investigator's Award from the Japanese Society for Xenobiotic Metabolism and Disposition in 1995, Young Investigator's Award from the Pharmaceutical Society of Japan in 1996, and AAPS Fellow in 2009. 